\title{
Relative Voltage Control of the Wind Farms Based on the Local Reactive Power Regulation
}

\author{
Yangyang Zhao *, Jianyun Chai * and Xudong Sun \\ State Key Laboratory of Control and Simulation of Power System and Generation Equipments, \\ Department of Electrical Engineering, Tsinghua University, Beijing 100084, China; Sunxd@mail.tsinghua.edu.cn \\ * Correspondence: zyy900318@126.com (Y.Z.); chaijy@mail.tsinghua.edu.cn (J.C.); \\ Tel.: +86-188-1136-3164 (Y.Z.); +86-10-6277-5559 (J.C.)
}

Academic Editor: Frede Blaabjerg

Received: 7 October 2016; Accepted: 22 February 2017; Published: 27 February 2017

\begin{abstract}
The fast and coordinated voltage control of distributed nodes inside a large scale wind farm is a stringent issue. To achieve the reactive power compensation without a dedicated upper communication system, this paper proposes a relative control strategy based on the local reactive power regulation. This method can realize the voltage coordination of the key equipment and feeder line nodes, on the basis of the relative voltage observations. Firstly, the stability analysis of a certain wind farm with pre-known parameters and structure is studied under the wind power fluctuations. Secondly, the optimal capacity configurations of the reactive power compensators are discussed, with multiple nodes inside the wind farms. Simulation results of a specific wind farm in North Hebei, China, validate the effectiveness of the proposed control, which is equipped with fast and stable voltage dynamic responses, as well as local reactive power compensations without remote communication.
\end{abstract}

Keywords: wind farm; relative voltage observation; distributed voltage control; local reactive power regulation

\section{Introduction}

Fluctuations of the distributed voltages and reactive power are one of the most common problems in the operation of large scale wind farms, as the internal voltages inside wind farms can be affected by the network parameters and wind speed under normal operations. The increasingly severe impacts of the voltage faults at the terminals of generators can lead to off-grid events of the wind generation system. Therefore, the stabilization of distributed voltages along feeder lines and the safety operation of wind farms in the weak AC grid have become major concerns on the autonomous energy internet.

Conventionally, centralized reactive power compensators are widely used at the point of common coupling (PCC) of the wind farms, such as a static var compensator (SVC), static synchronous compensator (STATCOM), etc. [1,2]. With the increasing amount of doubly-fed induction generator (DFIG) and permanent-magnet synchronous generator (PMSG) wind turbines installed in the wind farms, significantly more research has focused on the voltage regulations of the generator terminals and the local compensation of the distributed reactive power $[3,4]$. The hierarchical voltage control methods, based on the reactive power regulations of the variable-speed constant-frequency (VSCF) wind turbines, have been discussed in [5-7]. The coordinated control of centralized compensators and distributed wind generators were studied in [8,9]. Moreover, the multi-objective optimization models, including wind generators, STATCOM, and on load tap changing (OLTC) devices, were proposed in [10]. Most of the proposed reactive power compensation methods are based on the absolute voltage error or the fixed power factor, which are similar to the compensation strategy of the conventional generators. It is noted that all of the voltage control methods that are discussed in this paper are 
applied under normal operations of the wind farm, to maintain the stability of the internal voltages on the terminals of key equipment and feeder lines. This is different to low voltage ride-through (LVRT) methods, which are only applied under fault operations.

However, in contrast to the conventional power plant, the large scale wind farm is composed of various wind generators, whose rated output power is much smaller than the synchronous generators which are connected to the grid. Also, the reactive power capacity of the generation unit inside the wind farm is far less than the reactive power deficit when the grid voltage fault occurs. Thus, the reserved reactive power capacity of each unit cannot always maintain its terminal voltage at a constant value. Moreover, the power flow analysis of the wind farm mainly uses the algebraic accumulation of the reactive power, which is provided from compensation units as the equivalent compensated reactive power at the PCC [11,12]. However, the changes of the voltage phase angle along the feeder lines cannot be neglected, as the voltage distributions inside the wind farm are mainly caused by the inductance of the network. Also, as the wind farm always covers a wide area with scattered wind generators, the distribution features of the network are no longer negligible. Compared with the voltage regulation time of the power system, which is generally within tens of milliseconds, the consumed time for the conventional voltage control under master-slave mode would be greatly increased to the level of milliseconds. Therefore, it would further exacerbate the stability of the system's voltage, and even lead to power oscillations inside the wind farm. Therefore, the power flow analysis and voltage regulations inside the wind farm remain stringent issues [13].

In order to improve the dynamic performance of the internal voltage regulation inside the wind farm, as well as realize the local reactive power compensations without remote communication, the approach in this paper proposes compensating the reactive power based on the observations of local relative voltage errors. Firstly, the transfer functions of the reactive power and distributed voltages were analyzed, considering the changes in the voltage phase angle and fully network parameters. Then, observations of the local relative voltage errors are proposed, on the basis of the known constructions and parameters. Based on this method, the transfer characteristic of the voltages could enable every compensation node to track the voltage amplitude of the previous node all the way up to the PCC, without the need for centralized reactive power commands. Moreover, the optimal configuration for each compensator is discussed in relation to the limited reactive power capacity of the wind farm. Additionally, the proposed method could improve the voltage regulation time and system stability, without requiring communication between each compensator.

The rest of the paper is organized as follows. The distributed voltage transfer functions are outlined in Section 2. The coordinated voltage control, based on the local reactive power compensation, is provided in Section 3. The stability analysis of such a voltage control method is discussed in Section 4, and the simulation results for the wind power in North Hebei, China, are elaborated in Section 5, with conclusions drawn in Section 6.

\section{Distributed Voltage Transfer Functions}

The basic construction types of wind farms include radial structure, unilateral ring structure, bilateral ring structure, and composite ring structure. As a radial pattern has the advantages of a simple construction, convenient operation, and low cost, it has been widely used in wind farms across China, as shown in Figure 1.

As can be seen for the typical radial structure shown in Figure 1, the terminal voltage of the wind generator is usually set at $690 \mathrm{~V}$, and is boosted to the $35 \mathrm{kV}$ branch lines through its box transformer. The scattered generation units converge to the stem lines, and inject the active and reactive power to the $220 \mathrm{kV}$ grid through the main transformer at the PCC. A static var generator (SVG) or a SVC is always connected to the PCC, to regulate its voltage at the required base voltage value. Moreover, as the distributed voltages may fluctuate along the feeder lines, and in order to confine the terminal voltage of the generator within a safety operation margin, the reactive power compensators could be installed at the key confluence nodes. 


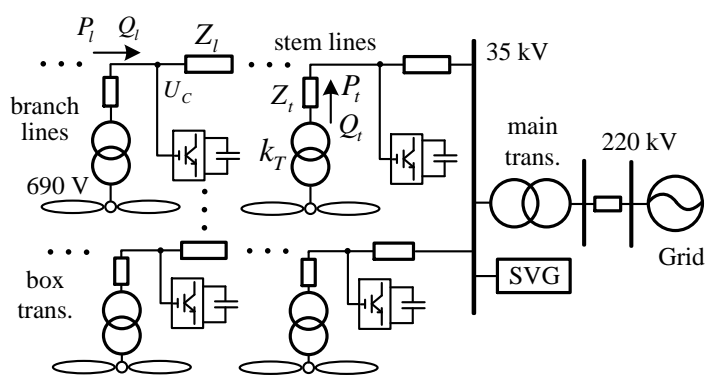

Figure 1. Radial structure of wind farm.

\subsection{Mathematical Model}

Considering the feeder lines are generally less than $100 \mathrm{~km}$, the capacitance effect of the transmission line can be neglected. The parameters of the branches and stems can be expressed as $R_{l}+j X_{l}$. Additionally, when the excitation impedance is comparatively larger than the leakage impedance, the parameters of the transformer can be expressed as $R_{\mathrm{t}}+j X_{\mathrm{t}}$. The nominal voltage is set as the nominal voltage of the PCC. A typical voltage transfer function between adjacent nodes is expressed in Equation (1). Assuming that the impedance between the adjacent nodes $\mathrm{A}$ and $\mathrm{B}$ is $R+j X$, the injected power from $\mathrm{A}$ to $\mathrm{B}$ is $P+j Q$.

$$
U_{a} \angle \theta_{a}=\left(U_{b}+\frac{P R+Q X}{U_{b}}+j \frac{P X-Q R}{U_{b}}\right) \angle \theta_{\mathrm{b}}
$$

where $U_{a}, \theta_{a}$ and $U_{\mathrm{b}}, \theta_{\mathrm{b}}$ are the magnitudes and phase angles of the nodes $\mathrm{A}$ and $\mathrm{B}$, respectively. The voltage drop in phase with $U_{\mathrm{b}}$ is the direct voltage, and the voltage drop with a 90 degree lag is the quadrature voltage. It is clear that the phase angles of the voltages vary along the feeder lines, and instead of applying the conventional model by algebraically accumulating the distributed reactive power at the PCC, the distributed voltages can be obtained by the iterations from the generation units to the branches and stems, up until the PCC. According to Equation (1), the ratio between the direct voltage and quadrature voltage is defined by the short-circuit ratio and impedance ratio. When $U_{\mathrm{b}}$ and the power flow from A to B are both 1 p.u., the voltage error of $U_{a}$ is calculated by neglecting the direct voltage and quadrature voltage, respectively. Moreover, the ratios of these two errors are shown in Figure 2, with the short-circuit ratio varying from $0 \%$ to $20 \%$, and the impedance ratio varying from $0.1 \%$ to $50 \%$.

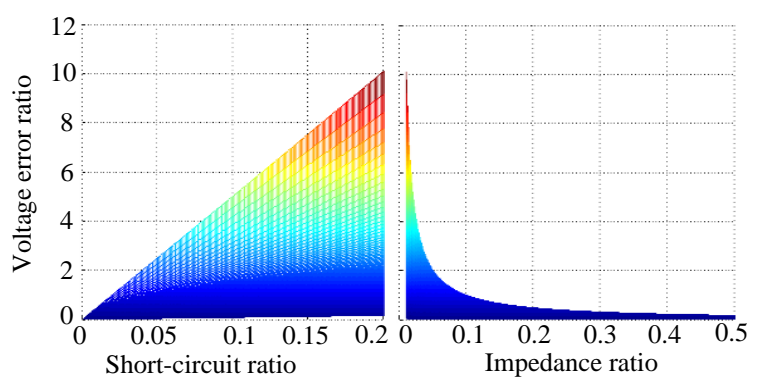

Figure 2. Voltage error ratio under different short-circuit ratios and impedance ratios.

If the ratio is between 0.1 and 10 , it means that the voltage drops of both the direct and quadrate axis are of the same scale, and none of them can be neglected. If the ratio is larger than 10, the quadrate voltage is negligible, while if it less than 0.1 , the direct voltage is negligible. Considering the variation ranges of the real parameters, the short-circuit ratio is about $0.5 \%$ to $1 \%$, and the impedance ratio is around 0.1 to 0.4 for the feeder lines in the wind farms. When considering the transformers, the short-circuit ratio is about $4 \%$ to $8 \%$, and the impedance ratio is around 0.02 to 0.05 [14,15]. 
The principle of reactive power compensation comprises the use of the voltage item $Q X / U_{b}$, which is generated from the reactive power through the inductance, to compensate the item $j \cdot P X / U_{b}$, generated by the variation of active power. The feature of the low impedance ratio enables the small reactive power to compensate for a larger voltage drop, caused by the variations of active power. It can be concluded from Figure 2 that the item of reactive power in the quadrate voltage can be neglected under most circumstances. In sum, the simplified transfer functions between adjacent nodes in the wind farm can be expressed as:

$$
U_{a} \angle \theta_{a}=\left(U_{b}+\frac{P R+Q X}{U_{b}}+j \frac{P X}{U_{b}}\right) \angle \theta_{\mathrm{b}}
$$

The feasibility and accuracy of the proposed methodology are based on the parameters of cable lines, and the detected voltage and current. Firstly, the accuracy of the voltage and current data is confined by the sampling precisions, which are usually within $1 \%$ for the common sensors. Then, for the cable lines and transformers, the parameters are basically constant with the same line type, physic material, and operation frequency. Furthermore, for the transformers, the data of the short circuit ratio, no-load current, and load loss, are also tested and provided by the manufacturers. After the construction of the wind farm, the length and positions of all of the cables and transformers are fixed. Thus, the parameters are reachable under normal operations.

\subsection{Objectives and Constrains of the Voltage Control}

The control objectives consist of the voltage of PCC and the terminal voltages of wind generators. The PCC voltage can be affected by a change of wind power, grid voltage disturbances, and as the reference voltage of the wind farm, the stability of the PCC voltage is of great importance. Meanwhile, as the generators need to send the wind power to the grid through a series of transformers and feeder lines, the variations of wind power could change the voltage distribution feature along the transmission paths, thus inducing fluctuations of the generator's terminal voltage. The stability of the terminal voltage ensures that the generation unit does not off-grid. The constraint function of the voltages can be written as:

$$
U_{\text {Cref }}-U_{\text {Cerr }} \leq U_{\mathrm{C}} \leq U_{\text {Cref }}+U_{\text {Cerr }}
$$

where $U_{C}$ is the voltage of the PCC or terminals of the generators, $U_{\text {Cref }}$ is the reference voltage, and $U_{C \text { Cerr }}$ is the error limit. These objectives can be achieved by the compensators installed at the PCC or key node along the feeder lines. To ensure the reliable operation of the compensators, the margin indices of the reactive power $\Delta Q_{C i}^{2}$ and voltage $\Delta U_{G i}^{2}$ are taken into consideration, as:

$$
\Delta Q_{C i}^{2}=\left(k_{Q} \frac{Q_{C}-Q_{C \text { ref }}}{Q_{C \max }-Q_{C \min }}\right)^{2}, \quad \Delta U_{G i}^{2}=\left(k_{U} \frac{U_{G}-U_{G \text { ref }}}{U_{G \max }-U_{G \min }}\right)^{2}
$$

where $Q_{C}$ and $Q_{C \text { ref }}$ are the present value and reference of the reactive power, respectively, and $Q_{C \max }$ and $Q_{C \min }$ are the reactive power limits of the compensators. $k_{Q}$ is the weight coefficient of the reactive power. Moreover, the voltage function has a similar definition for each corresponding symbol. It can be seen that the limits of the reactive compensator can determine whether the voltage requirements can be maintained. Considering the capacity of the grid is usually much larger than the wind farm, the voltage fluctuations caused by the grid cannot always be fully compensated by the wind farm, when the deficit exceeds the limits of all the reactive power compensators. Under such circumstances, the voltage loops are operated at the saturation mode, which leads to an uncontrollable state and exacerbates the voltage stability of a series of nodes inside the wind farm. Therefore, a relative voltage control, based on the local reactive power regulation, is proposed. 


\section{Relative Voltage Control Based on the Local Reactive Power Regulation}

\subsection{Observation Methods of the Relative Voltage}

The relative voltage is the voltage of the previous adjacent node along the feeder lines in the wind farm; as shown before, it can be defined by $U_{b} \angle \theta_{\mathrm{b}}$ in Equation (1). Considering that the levels of reactive power capacity are significantly different for the grid, the wind farm, and the wind generation unit, the voltage control should follow the local compensation principle. Based on the voltage transfer function in Equation (2), the voltage of the adjacent node can be derived from the voltage of the local node and the feeder line parameters. When the reactive compensator is installed at the key node on the feeder lines, this value can be served as the reference voltage of the local node, as:

$$
U_{C \mathrm{n}}=U_{\mathrm{Cref}}=\left[\left(U_{C}-\frac{R_{n} P_{n}+X_{n} Q_{n}}{U_{C}}\right)^{2}+\left(\frac{X_{n} P_{n}}{U_{C}}\right)^{2}\right]^{0.5}
$$

where $U_{C}$ is the sampled local voltage, $P_{n}, Q_{n}$ are the active power and reactive power flowing into the next node, respectively, and $Z_{n}=R_{n}+j X_{n}$ is the impedance of the power transmission path. Similarly, when the compensator is installed at the low-side of the transformer, the reference voltage can be given as:

$$
U_{\text {Tref }}=\frac{\left[\left(U_{T}^{*}-\frac{R_{\mathrm{t}} P_{\mathrm{t}}+X_{\mathrm{t}} Q_{\mathrm{t}}}{U_{T}^{*}}\right)^{2}+\left(\frac{X_{\mathrm{t}} P_{\mathrm{t}}}{U_{T}^{*}}\right)^{2}\right]^{0.5}}{\mathrm{k}_{T}}
$$

where $U_{T}^{*}$ is the sampled voltage of the high-side of transformer, $\mathrm{k}_{T}$ is the turns ratio, $P_{\mathrm{t}}, Q_{\mathrm{t}}$ are the power flowing out the transformer, and $Z_{\mathrm{t}}=R_{\mathrm{t}}+j X_{\mathrm{t}}$ is the impedance from the transformer to the nearest confluence node. Considering the parameters are roughly constant after the wind farm has been built, the items that are required to generate the reference voltage are locally observable. Theoretically, all the voltages of the key nodes, equipment, and generators, can be maintained at the same level inside the wind farm, based on such a reactive power compensation method. Furthermore, this strategy can eradicate the need for long distance communication and complex scheduling, so as to achieve a fast and coordinated voltage control with local observations. On account of the reference voltage set as the observed voltage of the previous node, all the voltages of the key nodes inside the wind farm are controlled as the same voltage value of PCC, even when it deviates from its nominal value. This relative voltage control strategy can assure that the compensators do not exceed the reactive power capacity during regulation, and the PCC voltage can be set back to a nominal value through secondary control, such as OLTC.

\subsection{Local Reactive Power Regulation Methods}

The proposed relative voltage is set as the reference voltage, and the result of the voltage control loop operates as the reactive power reference of the current control loop. The final reference voltage signal for the pulse-width modulation (PWM) module of the converter is expressed as:

$$
U_{\mathrm{C}}=\left[\left(U_{\mathrm{ref}}-U_{\mathrm{o}}\right)\left(K_{\mathrm{pU}}+\frac{K_{\mathrm{iU}}}{s}\right)-I_{\mathrm{o}}\right] \cdot\left(K_{\mathrm{pI}}+\frac{K_{\mathrm{iI}}}{s}\right)
$$

where $U_{\text {ref }}$ is the proposed relative voltage, $U_{\mathrm{o}}$ is the sampled voltage, $I_{\mathrm{o}}$ is the sampled reactive current, and $K_{\mathrm{pU}}, K_{\mathrm{iU}}, K_{\mathrm{pI}}$, and $K_{\mathrm{iI}}$ are the proportional-integral (PI) parameters of the voltage loop and current loop, respectively.

From the perspective of the wind farm, if all of the junction nodes can realize the proposed local reactive power regulation, the voltages inside the wind farm are globally maintained, in accordance with the PCC voltage. The control diagram shown in Figure 3 elaborates the local reactive power control strategy inside the wind farm. The compensation methods are mainly divided into three sections, including box transformer compensation, feeder lines compensation, and PCC compensation. Transformer compensation regulates the voltage drop in the leakage impedance of the box transformer, 
the feeder lines compensation regulates the voltage drop between adjacent confluence nodes, and the PCC compensation regulates the voltage drop in the main transformer.

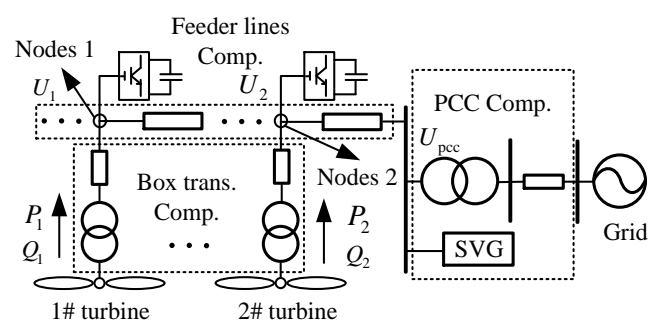

Figure 3. Voltage control diagram of wind farm.

Considering that the capacity of the installed compensators and the construction costs of a wind farm are limited, the global voltage control of all the confluence nodes along the feeder lines and terminals of the generators, cannot be fully achieved. Then, the voltage margin index and reactive power margin index are used to form the optimization functions:

$$
\min f(x)=\alpha_{\mathrm{Q}} \sum_{i=1}^{N_{C}} \Delta Q_{C i}^{2}+\alpha_{U} \sum_{i=1}^{N_{G}} \Delta U_{G i}^{2}
$$

where $\alpha_{\mathrm{Q}}, \alpha_{\mathrm{U}}$ are the weight coefficients of the reactive power index and voltage index, respectively, $N_{C}$ is the number of compensators, and $N_{G}$ is the number of wind turbines. An interior point filter (IPFA) optimization algorithm is applied on account of its good convergence, fast optimization speed, and strong robustness $[14,15]$. Thus, the local reactive power regulation can still be realized with a limited capacity of the compensators, controlling the voltages inside the wind farm within the required bound. Compared to the centralized compensation methods, the proposed local compensation strategy can theoretically realize the global voltage control with distributed compensators of small reactive power capacity, though the maintenance fee and construction cost slightly increase.

The proposed relative voltage control method would solve the problem of reactive power limitation when the voltages are regulated to meet the absolute reference value, because the capacity of the distributed generation unit is far less than the grid deficit. Also, the reactive power can be compensated locally, without communication from the upper calculation center, which delays the control speed. On the basis of what has been discussed above, the stability of such control methods is discussed below.

\section{Stability Analysis of the Voltages inside the Wind Farm}

The proposed relative voltage control can theoretically coordinate all the voltages of the wind farm through local reactive power control. However, such distributed control, which involves multiple compensation nodes, would not easily reveal the stability and dynamic performance. Thus, the stability analysis is further studied in this section. As the regulations are achieved by the PI controllers embedded in the distribution compensators, the stability of these control loops can affect the stability of the wind farm. Stability analysis is based and applied on the system consisting of two generators, as shown in Figure 3. Assuming the voltage of the PCC is constant, the output active power of the $1 \#$ wind generator $P_{1}$ is set as the disturbance, and the voltage of the $2 \#$ wind generator $U_{2}$ is set as the observation. Considering that the voltage transfer function has the quadric expressions of the disturbance variables, the stability analysis is initially studied based on linearized equations, and then its performance is analyzed by using the local linearization. The voltage relationship and reactive power equations are expressed as: 


$$
\begin{gathered}
U_{2}=U_{\mathrm{pcc}}+\left[\left(P_{1}+P_{2}\right) R+\left(Q_{1}+Q_{2}\right) X\right] / U_{\mathrm{pcc}} \\
U_{1}=U_{2}+\left(P_{1} R+Q_{1} X\right) / U_{\mathrm{pcc}} \\
Q_{1}=\left(U_{2}-U_{1}\right)\left(\mathrm{K}_{P 1}+\frac{\mathrm{K}_{\mathrm{II}}}{\mathrm{s}}\right) \\
Q_{2}=\left(U_{\mathrm{pcc}}-U_{2}\right)\left(\mathrm{K}_{P 2}+\frac{\mathrm{K}_{\mathrm{I}}}{\mathrm{s}}\right)
\end{gathered}
$$

where the variables are in accordance with Figure 3, and $\mathrm{K}_{\mathrm{P} 1}, \mathrm{~K}_{\mathrm{I} 1}$ and $\mathrm{K}_{\mathrm{P} 2}, \mathrm{~K}_{\mathrm{I} 2}$ are the PI parameters of the installed compensators in Node 1 and Node 2, respectively. Then, the transfer function from $U_{2}$ to $P_{1}$ can be obtained by iterating Equation (9).

$$
\frac{U_{2}(s)}{P_{1}(s)}=\frac{R s^{2}}{\left(s+X K_{\mathrm{P} 2} s+X K_{\mathrm{I} 2}\right)\left(s+X \mathrm{~K}_{\mathrm{P} 1} s+X \mathrm{~K}_{\mathrm{I} 1}\right)}
$$

The dominant poles of the transfer function are $\omega_{1}=-\mathrm{XK}_{\mathrm{I} 1} /\left(1+\mathrm{XK} \mathrm{P}_{\mathrm{P}}\right), \omega_{2}=-\mathrm{XK}_{\mathrm{I} 2} /\left(1+\mathrm{XK}_{\mathrm{P} 2}\right)$. As the parameters of the impedance and PI controllers are all positive, the poles are located in the negative half of the plane, which shows the global stability of the system. Moreover, when applying the whole nonlinear function as Equation (2), the $U_{2}$ can be expressed in the second-order form, with a voltage of PCC that is equal to the nominal value:

$$
\frac{U_{2}}{U_{\mathrm{pcc}}}=1+\left(P_{1}+P_{2}\right) \mathrm{R}+\left(Q_{1}+Q_{2}\right) X+P_{1}^{2} \frac{X^{2}}{2}+P_{2}^{2} \frac{X^{2}}{2}+P_{1} P_{2} X^{2}
$$

Then, the equation is expanded and locally linearized at the operation point $P_{1}=0$, as follows:

$$
\frac{U_{2}(s)}{P_{1}(s)}=\frac{s\left(\mathrm{~K}_{\mathrm{P} 1} P_{2} X^{3} s+P_{2} X^{2} s+R s+\mathrm{K}_{\mathrm{I} 1} P_{2} X^{3}\right)}{\left(s+X \mathrm{~K}_{\mathrm{P} 2} s+X \mathrm{~K}_{\mathrm{I} 2}\right)\left(s+X \mathrm{~K}_{\mathrm{P} 1} s+\mathrm{XK}_{\mathrm{I} 1}\right)}
$$

Compared with the linearized transfer function, the dominating poles of the local linearized function are still located in the left half of the plane. Then, the perturbation observations under different initial operation points of $P_{1}(0)$, are shown in Figure 4 .

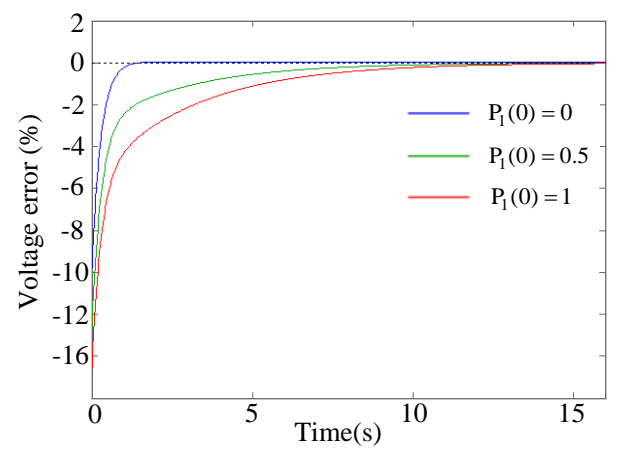

Figure 4. Voltage step responses of a nonlinear function.

From the figures above, it is clear that the step responses of $U_{2}$ are convergent when $P_{1}(0)$ is set as $0,0.5$, and 1 , respectively. Thus, the voltages inside the wind farm, which obey the nonlinear transfer function, can still have a fairly big stability margin under the proposed relative voltage control. However, the stability of each node voltage under the conventional voltage control based on the absolute value, is constrained by the upper centralized controller which needs to sample, calculate, and transfer a huge amount of data from the distributed wind generation unit and various nodes on the feeder lines. Under the large disturbance from grid-side or turbine-side, the transmission speed of the instructions for the voltage and reactive power usually takes tens of milliseconds, while the voltage control speed for the grid is usually at the level of milliseconds, and the oscillations of voltage 
and reactive power frequently occur. The proposed relative voltage control equips the reactive power compensations with fast and stable features, which are highly valued in practical application.

\section{Simulation Results}

The parameters and constructions of the simulation model are obtained from a specific wind farm in North Hebei, China, as is shown in Figure 1. The whole installed capacity of the wind farm is $49.5 \mathrm{MVA}$, which includes 33 wind generation units. The parameters of the feeder lines, main transformer, and box transformers, are elaborated in the appendix. The traditional wind farm has a radial structure consisting of three feeder lines, A, B, and C, which lie parallel at the PCC. Each of these feeder lines has 12,12 , and nine wind turbines connected in series, respectively. As the voltages of the PCC determine the base value of all the voltages inside the wind farm, and the terminal voltages of generators assure the safety operation and grid-connected mode of generation units, the priority of these voltages should also be taken into consideration. To elaborate the effects of the proposed voltage control methods, they can be further divided into four categories, represented as S1 to S4, while considering the voltage priorities. These control strategies are all compared with the conventional absolute voltage value control, which is represented by Conventional in the figures.

(1) Relative voltage control is applied at the PCC (S1).

(2) Relative voltage control is applied at the PCC and the terminals of the generators (S2).

(3) Relative voltage control is applied at the PCC, the terminals of the generators, and the key nodes along the feeder lines (S3).

(4) Relative voltage control is applied at the locations as category 3 under a limited reactive power capacity (S4).

\subsection{Relative Voltage Control at PCC of the Wind Speed}

A typical daily active wind power curve of the wind farm in North Hebei is set as the active output power at the PCC, which is shown in Figure 5. In order to reflect the difference between the absolute voltage value control and relative voltage control, the grid voltage is fixed at 0.98 .

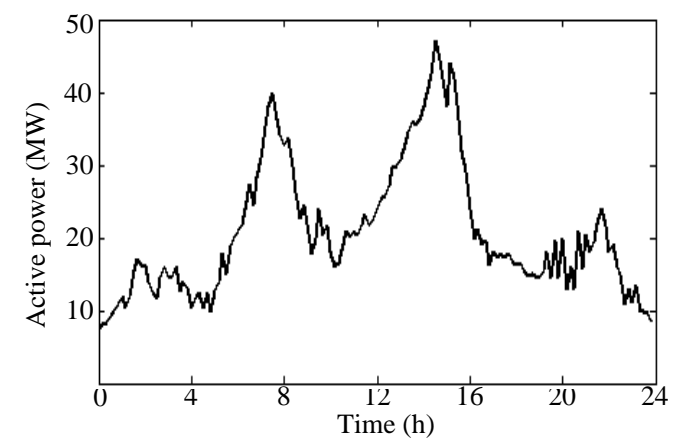

Figure 5. Daily active power curves at point of common coupling (PCC) of the wind farm.

The absolute value of the PCC voltage is used in the conventional reactive compensation strategy. The PCC voltage reference value in this method is usually given according to the system schedule set to the 1 per-unit (p.u.) in the simulation. Figure 6a shows the comparisons of reactive power compensation under absolute voltage control and relative voltage control, at the PCC point. It can be seen from Figure $6 a$ that if the wind farm only needs to meet the voltage requirement at the PCC, then control strategy 1 can cause the wind voltage to fluctuate slightly, with the grid voltage under normal operating conditions. The voltage control of the wind farm would only compensate for the voltage drop caused by the active fluctuations of the wind generators, and would not actively support the reactive power loss of a grid with a higher capacity level. Therefore, the reactive power 
compensation is more effective than the traditional absolute voltage compensation. While the absolute voltage control may cause oscillations when the grid voltage deviates from the rated value, the reactive power compensators cannot be compared with the grid capacity and this demonstrates its limitation. In this paper, the reactive power capacity and voltage margins of the wind farm taken into account the relative voltage control, which is more reasonable than the traditional absolute voltage control.

When the wind farm requires the global control of the voltage, it is necessary to assemble the terminals of the generators and the key confluence nodes with the reactive power compensators. The conventional method needs to realize the reactive power schedule through the centralized control calculation, and the updating cycle of the instructions takes about $80 \mathrm{~ms}$ due to the long distance and huge data transmission, while the proposed strategy S3 can increase its control cycle to $3 \mathrm{~ms}$ for it is based on local parameters and observed signals. The PCC voltage fluctuations of the above two strategies are shown in Figure 6b, and the voltage level is set at $35 \mathrm{kV}$ (1.0 p.u.).

It can be seen that it is necessary to install the distributed compensators at all key nodes to realize the global voltage control of the wind farm. Also, the PCC voltage under the conventional control method will fluctuate more drastically, due to the delay of the control signal, and the local reactive power compensation based on the relative voltage observation can realize the global control of the wind farm, without centralized dispatching. This demonstrates a strong advantage in terms of faster compensation and more stable voltage regulation.

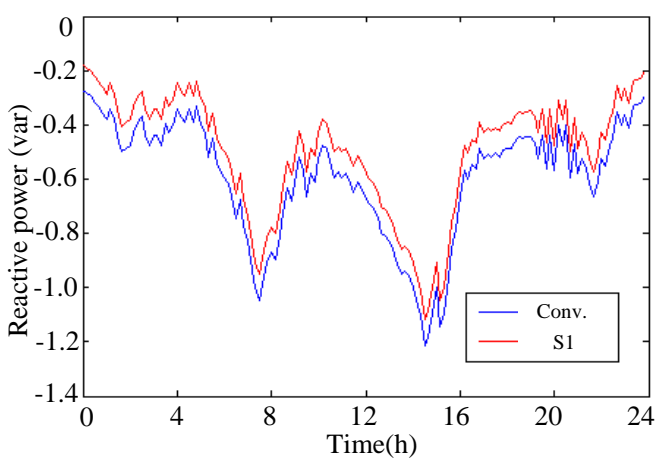

(a)

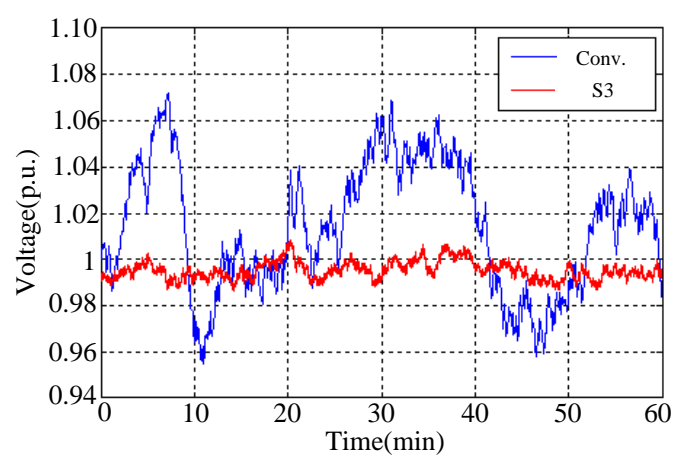

(b)

Figure 6. (a) Reactive power and (b) voltage curves at the PCC under different control methods.

\subsection{Voltage Distributions of the Wind Farm}

Assuming that each generation unit outputs $60 \%$ of its rated power, and the grid voltage is set as 0.98 p.u., the voltage of each node and the corresponding reactive power output are shown in Figure 7a,b, respectively. Taking into account the wind farm voltage fluctuation requirements and the limitation of the reactive power compensators, control strategy S4 provides the optimized solutions for the reactive power compensations under limited capacity. The node numbers are arranged according to their electrical distances from the PCC point, where the PCC node is No. 34, and the node located the furthest away is No. 1. Though the VSCF turbines can also be applied to compensate for the voltages, the reactive power capacity is always limited. As different relative voltage control strategies require different amounts of reactive power from the turbines, the attached STATCOMs are applied at the turbine-side to avoid the lack of the reactive power capacity in the simulation.

As the conventional control and proposed strategy S1 only regulate the PCC voltage, the reactive power distribution diagram in Figure $7 \mathrm{~b}$ elaborates the performance of the rest of the strategies from S2 to S4, which all have the distributed compensators installed at the voltage nodes from No. 1 to No. 34. It is clear that the reactive power of the compensators is effectively decreased while applying the proposed relative voltage control. Thus, in a way, the VSCF turbine can be applied to maintain 
the terminal voltage, without fear that the required reactive power will exceed its limited reactive power capacity.

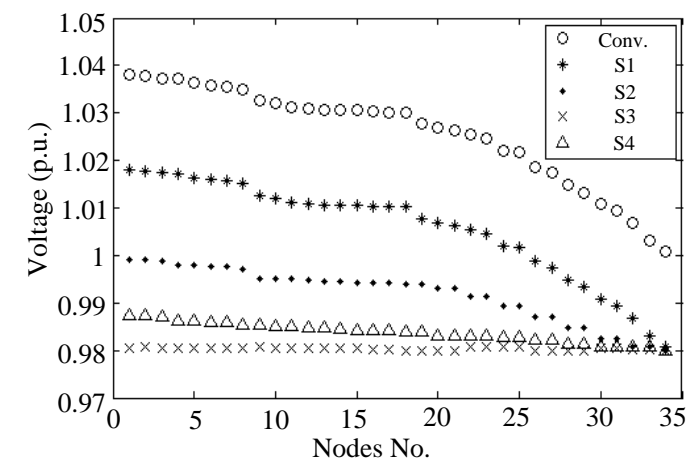

(a)

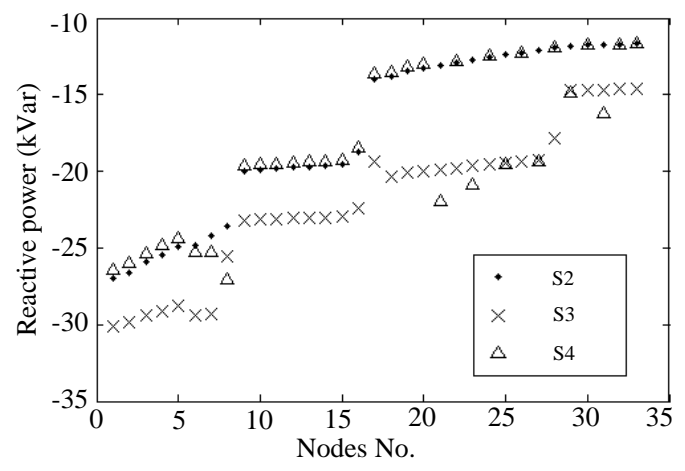

(b)

Figure 7. (a) Voltage and (b) reactive power distributions of the wind farm under different control methods.

The compensated reactive power at the PCC needs -0.79123 Mvar under the conventional method, -0.68672 Mvar under the S1 method, -0.21871 Mvar under the S2 method, -0.16873 Mvar under the S3 method, and -0.2102 Mvar under the S4 method. According to the unit price of the compensators in the market, the average cost for the complete set of reactive power compensation equipment is about $80,000 ¥ /$ Mvar, which also includes the initial costs of the power converters. Table 1 shows the total amount of reactive power equipment and investment required for different control strategies.

Table 1. Required total reactive power compensators and investment of different control modes.

\begin{tabular}{cccccc}
\hline Control Methods & Conventional & S1 & S2 & S3 & S4 \\
\hline Total reactive power (Mvar) & -0.79123 & -0.68672 & -0.79340 & -0.86184 & -0.83034 \\
Total investment $\left(10^{4} ¥\right)$ & 6.32984 & 5.49376 & 6.34720 & 6.89472 & 6.64272 \\
\hline
\end{tabular}

To sum up the different control methods above:

(1) For the control of the PCC voltage, it can be maintained at the per-unit value of 1 based on the absolute voltage. The strategy S1 of the relative voltage control sets the PCC reference voltage according to the present grid voltage value. Considering the limited capacity of the wind farm, the required compensation power of S1 is smaller than the conventional method. However, these two methods cannot effectively regulate the global voltage inside the wind farm, due to lack of distributed reactive power compensators. Under such circumstances, the maximum voltage error inside the wind farm under the conventional method is 3.8\%.

(2) Strategy S2 can compensate for the voltage drop in the box transformers, based on the local observed signals. The distributed reactive power is injected into the PCC, and therefore, the capacity of the PCC compensator can be greatly reduced. However, the voltage drops along the feeder lines are still not compensated for. Under this strategy, the maximum voltage deviation within the wind farm is $1.9 \%$.

(3) Strategy S3 can realize the global voltage controllability with compensators installed at all of the key voltage nodes. Therefore, all the nodes inside the wind farm can be maintained as the PCC voltage in per-unit value, which increases the stability of the terminal voltage of the generators, so as to reduce the off-grid accidents. Since the voltage drops along the feeder lines are also compensated for, the total reactive power capacity will increase. With each distributed unit 
that can compensate for the local relative voltage loss, the reactive power required at the PCC is further reduced compared to strategy S2. Compared to the existing global voltage control strategy, based on upper scheduling, the stability and dynamic response are much improved.

(4) Strategy S4 applies the IPFA algorithm to realize the optimized compensation under the reactive power capacity. Because the structure and parameters of the wind farm are relatively fixed, the optimization method can be given in advance. In the simulation, the maximum voltage error can be regulated within $1 \%$, which can also satisfy the optimal reactive power capacity margin.

It can be seen from the parameters in the appendix that the length of three feeder lines are close to each other, and each line contains three major confluence nodes which are farther apart. Therefore, the voltages are mainly divided into three groups by the confluence section. It is clear that, in the optimization results, the installed compensators were located at the main confluence nodes, to reduce the average errors of the distributed voltages. As the time constant of the voltage variations requests faster reactive power compensations, the proposed relative voltage regulation strategy, based on the local observed information, is superior to the conventional method with respect to the dynamic response and stability.

\section{Conclusions}

Considering that the increasingly deteriorative voltage dynamic response and stability problems of wind farms, due to the present reactive power compensations, always involve a large data scheduling and transmission delay, this paper proposed a relative voltage coordination method based on the local reactive power compensations. As the total power capacity of the wind farm is far less than the grid, this method only compensates for the local reactive power caused by the active power fluctuations from the wind turbines, which reduces the required reactive power from each compensator compared with the absolute voltage value regulation, and further avoids the possible power oscillation caused by the clipped controllers. Secondly, as the network parameters are pre-known, and the local voltage and current information can be observed locally, the proposed strategy can realize the global voltage control and reactive power allocation without an upper calculation center, as well as provide a faster and more stable dynamic response without long distance intercommunication. Moreover, the optimization algorithm can also be applied in the proposed control, to regulate the maximum voltage error of the wind farm within the requirements of the minimum reactive power capacity. Finally, a simulation example of a wind farm in northern Hebei proved the effectiveness of the proposed voltage coordination control, which improves the dynamic performance of the internal voltage regulation inside the wind farm, as well realizing the local reactive power compensations without remote communication.

Acknowledgments: This paper is supported by National Natural Science Foundation of China (NSFC) under Grant No. U1510208.

Author Contributions: Yangyang Zhao and Jianyun Chai conceived and designed the control strategy; Yangyang Zhao realized the operation of the system; Xudong Sun contributed to the application of the analysis tools and provided helpful advices; Yangyang Zhao wrote the paper.

Conflicts of Interest: The authors declare no conflict of interest.

\section{Appendix}

The parameters of the feeder lines and transformers are shown in the following tables (Tables A1-A3). 
Table A1. Information of feeder lines in wind farm.

\begin{tabular}{|c|c|c|c|c|c|}
\hline Nodes No. & Length from PCC (km) & Feeder Lines No. & Nodes No. & Length from PCC (km) & Feeder Lines No. \\
\hline 1 & 20.671 & A & 18 & 13.026 & C \\
\hline 2 & 20.367 & A & 19 & 10.851 & A \\
\hline 3 & 19.507 & B & 20 & 10.325 & A \\
\hline 4 & 19.084 & B & 21 & 9.815 & A \\
\hline 5 & 18.658 & B & 22 & 9.628 & A \\
\hline 6 & 18.217 & B & 23 & 8.912 & A \\
\hline 7 & 18.132 & C & 24 & 8.457 & A \\
\hline 8 & 18.012 & C & 25 & 8.121 & B \\
\hline 9 & 15.960 & A & 26 & 7.561 & B \\
\hline 10 & 15.573 & A & 27 & 7.098 & B \\
\hline 11 & 15.238 & A & 28 & 6.549 & B \\
\hline 12 & 15.001 & A & 29 & 6.159 & B \\
\hline 13 & 14.565 & B & 30 & 5.358 & C \\
\hline 14 & 14.321 & B & 31 & 5.098 & C \\
\hline 15 & 13.984 & B & 32 & 4.897 & C \\
\hline 16 & 13.601 & C & 33 & 4.671 & C \\
\hline 17 & 13.235 & C & & & \\
\hline
\end{tabular}

Table A2. Parameters of transformers in wind farm.

\begin{tabular}{cccccccc}
\hline Devices & $\begin{array}{c}\text { Rated Voltage } \\
(\mathbf{k V})\end{array}$ & $\begin{array}{c}\text { Rated } \\
\text { Current (A) }\end{array}$ & $\begin{array}{c}\text { Rated } \\
\text { Frequency (HZ) }\end{array}$ & $\begin{array}{c}\text { Short-Circuit } \\
\text { Ratio (\%) }\end{array}$ & $\begin{array}{c}\text { No-Load } \\
\text { Current (\%) }\end{array}$ & $\begin{array}{c}\text { Load Loss } \\
(\mathbf{k W})\end{array}$ & $\begin{array}{c}\text { No-Load } \\
\text { Loss (kW) }\end{array}$ \\
\hline Box transformer & $37 / 0.69$ & $25 / 1339$ & 50 & 6.5 & 0.2 & 183.609 & 36.66 \\
Main transformer & $220 / 37$ & $131 / 780$ & 50 & 10.5 & 0.07 & 13260 & 1650 \\
\hline
\end{tabular}

Table A3. Parameters of feeder lines in wind farm.

\begin{tabular}{cccccc}
\hline Devices & $\begin{array}{c}\text { Rated } \\
\text { Voltage (kV) }\end{array}$ & Line Type & $\begin{array}{c}\text { Resistance } \\
(\mathbf{p . u .} / \mathbf{k m})\end{array}$ & $\begin{array}{c}\text { Conductance } \\
(\mathbf{p u} / \mathbf{k m})\end{array}$ & $\begin{array}{c}\text { Admittance } \\
(\mathbf{p u} / \mathbf{k m})\end{array}$ \\
\hline Feeder lines & 35 & LGJ-400/25 & 0.00139 & 0.00765 & 0.00007 \\
\hline
\end{tabular}

\section{References}

1. Yang, S.; Wang, W.S.; Huang, Y.H.; Xu, X.Y.; Chai, H.D. Coordinated strategy for automatic voltage control of wind farm. In Proceedings of the 2012 China International Conference on Electricity Distribution (CICED), Shanghai, China, 10-14 September 2012.

2. Xu, L.; Yao, L.; Sasse, C. Comparison of using SVC and STATCOM for wind farm integration. In Proceedings of the International Conference on Power System Technology, Chongqing, China, 22-26 October 2006.

3. Xu, D.; Li, R.; Liu, Y.; Lang, Y. Reactive power analysis and control of doubly fed induction generator wind farm. In Proceedings of the 13th European Conference Power Electronics and Applications (EPE'09), Barcelona, Spain, 8-10 September 2009.

4. Tapia, G.; Tapia, A.; Ostolaza, J.X. Proportional integral regulator-based approach to wind farm reactive power management for secondary voltage control. IEEE Trans. Energy Convers. 2007, 22, 488-498. [CrossRef]

5. Moursi, M.E.; Joos, G.; Abbey, C. A secondary voltage control strategy for transmission level interconnection of wind generation. IEEE Trans. Power Electron. 2008, 23, 1178-1190. [CrossRef]

6. Masaud, T.; Sen, P.K. A comparative study of the implementation of STATCOM and SVC on DFIG-based wind farm connected to a power system. In Proceedings of the IEEE Power and Energy Society General Meeting, San Diego, CA, USA, 22-26 July 2012.

7. Guo, Q.; Sun, H.; Liu, Y.; Chen, R.; Wang, B.; Zhang, B. Distributed automatic voltage control framework for large-scale wind integration in China. In Proceedings of the IEEE Power and Energy Society General Meeting, San Diego, CA, USA, 22-26 July 2012.

8. Fakham, H.; Ahmidi, A.; Colas, F.; Guillaud, X. Multi-agent system for distributed voltage regulation of wind generators connected to distribution network. In Proceedings of the IEEE Power \& Energy Society (PES) Innovative Smart Grid Technologies Conference Europe (ISGT Europe), Gothenburg, Sweden, 11-13 October 2010. 
9. Dicorato, M.; Forte, G.; Trovato, M. Voltage compensation for wind integration in power systems. In Proceedings of the 3rd IEEE International Symposium Power Electronics for Distributed Generation Systems (PEDG), Aalborg, Denmark, 25-28 June 2012; pp. 464-469.

10. Chiandone, M.; Campaner, R.; Arcidiacono, V.; Sulligoi, G.; Milano, F. Automatic voltage and reactive power regulator for wind farms participating to TSO voltage regulation. In Proceedings of the PowerTech Eindhoven 2015 Conference, Eindhoven, The Netherlands, 29 June-2 July 2015.

11. Chen, H.F.; Qiao, Y.; Lu, Z.X. Study on coordinated voltage control strategy of DFIG wind farm. In Proceedings of the IEEE Power and Energy Society General Meeting, San Diego, CA, USA, 22-26 July 2012.

12. Bo, D.; Wenying, L.; Jiaming, W.; Gang, C.; Bolin, Z. The coordinating control measures of reactive power and voltage in the area with large-scale wind farms. In Proceedings of the 2nd International Articial Intelligence, Management Science and Electronic Commerce (AIMSEC) Conference, Jiaozuo, China, 8-10 August 2011; pp. 4423-4426.

13. Abdel-Aziz, E.Z.; Ishaq, J.; Al-Harthi, R. Impact of distributed generators on voltage stability in transmission network. In Proceedings of the Saudi Arabia Smart Grid (SASG), Jeddah, Saudi Arabia, 7-9 December 2015.

14. Yang, S.; Zhou, J.Y.; Li, Q.; Pan, Y.; Li, X.L. An interior-point algorithm for reactive power optimization. In Proceedings of the Asia-Pacic Power and Energy Engineering Conference (APPEEC), Wuhan, China, 25-28 March 2011.

15. Fan, Z.; Chang, X.; Wang, H.; Pu, T.; Yu, T.; Liu, G. Discrete reactive power optimization based on interior pointalgorithm and complementarity theory. In Proceedings of the International Power System Technology (POWERCON) Conference, Chengdu, China, 20-22 October 2014; pp. 210-214.

(C) 2017 by the authors. Licensee MDPI, Basel, Switzerland. This article is an open access article distributed under the terms and conditions of the Creative Commons Attribution (CC BY) license (http:/ / creativecommons.org/licenses/by/4.0/). 